\title{
Characterization of calcium phosphate deposited on valve metal by anodic oxidation with polarity inversion
}

\author{
Seigo OKAWA, Kikuo HOMMA, Mitsugu KANATANI and Kouichi WATANABE \\ Division of Biomaterial Science, Department of Oral Health Science, Niigata University Graduate School of Medical and Dental Sciences, 5274 \\ Gakkoucho-dori 2, Chuo-ku, Niigata, 951-8514, Japan \\ Corresponding author, Seigo OKAWA; E-mail: sokawa@dent.niigata-u.ac.jp
}

\begin{abstract}
Electrochemical deposition of calcium phosphate (CAP) on valve metals such as $\mathrm{Ta}, \mathrm{Nb}$, and $\mathrm{Zr}$, was performed by anodic oxidation with alternate polarity inversion at an applied 20 VDC. A saturated hydroxyapatite(HAP)-phosphoric acid solution (pH 3) was used as the electrolyte. FTIR, XRD, and XPS were employed to investigate the detailed characteristics of the deposition. HAP was precipitated on Ta; HAP including brushite and monetite on $\mathrm{Nb}$; and HAP and monetite on Zr. The Ca/P atomic ratios were 1.3-1.5 by XPS, and $\mathrm{HPO}_{4}{ }^{2-}$ bands were detected on Ta by FTIR. Therefore, the HAP precipitated on Ta was a Ca-deficient HAP. In addition, the XPS spectra of the specimens showed that phosphate ions were incorporated into the anodic oxide film. Deposits with nano-grain size were observed by AFM. The results confirmed that CAP with nano-grain size was deposited on valve metals by the anodic oxidation with polarity inversion.
\end{abstract}

Keywords: Calcium phosphate, Valve metal, Anodic oxidation

Received Dec 4, 2008: Accepted Apr 20, 2009

\section{INTRODUCTION}

Titanium and its alloys have been used for dental or orthopedic implants in the living body ${ }^{1)}$. Valve metals such as tantalum, zirconium, niobium and their alloys have attracted considerable attention as dental materials ${ }^{2}$. Since these metals and alloys have excellent corrosion resistance, they produce few allergic and toxicity reactions in the living body ${ }^{3}$. Therefore, these materials are known to have good biocompatibility and are usually used for dental or skeletal implants ${ }^{4)}$.

For these reasons, many researchers ${ }^{5-7)}$ reported that better osteoconductivity was obtained by surface treatments and modifications using chemical and physical methods. A plasma-spraying technique, which is most commonly used for coating hydroxyapatite (HAP) on metallic implants, was investigated and used to produce HAP coatings with high stability against mechanical fracture in the living body ${ }^{8}$. However, there is a possibility for HAP to decompose in the coatings, since the technique involves a high-temperature processing step ${ }^{8}$.

Ishizawa et al.9) have developed a new method for forming highly-crystallized HAP layers on $\mathrm{Ti}$ and Ti6Al4V alloy by anodic oxidation at 350 VDC and subsequent hydrothermal treatment. In their study, a HAP layer (5-30 $\mu$ m thickness) was synthesized on the metals. On the other hand, Ban et al. ${ }^{10)}$ reported that granular and needle-like calcium phosphate compounds (CAPs) were electrochemically deposited on a Ti plate. Monma ${ }^{11)}$ demonstrated that the electrochemical deposition of Ca-deficient apatite on a stainless steel substrate can be carried out in an electrolyte. In his experiment, $\mathrm{HAP}$ and brushite, $\mathrm{CaHPO}_{4} \cdot 2 \mathrm{H}_{2} \mathrm{O}$ (DCPD), or monetite, $\mathrm{CaHPO}_{4}$ (DCPA), were deposited on the metal. The shapes of the apatite deposits such as ellipsoidal grains, needles or fine granules depended on temperature and current density. Hanna et al. ${ }^{12)}$ reported that pure brushite layer (about $50 \mu \mathrm{m}$ thickness) can be electrodeposited on Ti alloy substrate and transformed to HAP by heat treatment. After transformation to HAP, crystal size and porosity, decrease with the appearance of some needle-shape crystals.

As mentioned above, the plasma-spraying technique and electrochemical precipitation of CAPs on $\mathrm{Ti}$ and stainless steal plates under appropriate experimental conditions are thus possible. Film thick of their productions is the order of micrometers. We focused on a new method in which CAPs were deposited by electrochemical surface treatment to coat a thin film coating. The research subjects of this work were to directly deposit CAPs on valve metals and to control the order of nanometer thickness of the deposition layer. We propose here a new precipitation method, that is, anodic oxidation with polarity inversion in HAP-phosphoric acid ( $\left.\mathrm{HAP}-\mathrm{H}_{3} \mathrm{PO}_{4}\right)$ solution, and characterize CAPs produced on valve metals by anodic oxidation with polarity inversion.

\section{MATERIALS AND METHODS}

\section{Preparation of specimens}

Valve metal of Ta(purity 99.95\%, Nilaco Co., Ltd., Tokyo, Japan), $\operatorname{Zr(purity~99.2\% ,~Nilaco~Co.,~Ltd.)~and~}$ $\mathrm{Nb}$ (purity 99.9\%, Nilaco Co., Ltd. ) were used in this study. Each valve metal was cut into disks of $15 \mathrm{~mm}$ diameter and $5 \mathrm{~mm}$ thickness. The disk specimens were mechanically abraded with water proof $\mathrm{SiC}$ abrasive papers up to \#800 grit under tap water. Using colloidal silica (Mastermet, Buehler, IL, USA), the abraded specimens were polished with a buffing cloth (Chemomet I, \#40-7908, Buehler). Both abrading and polishing were conducted on a polishing machine 


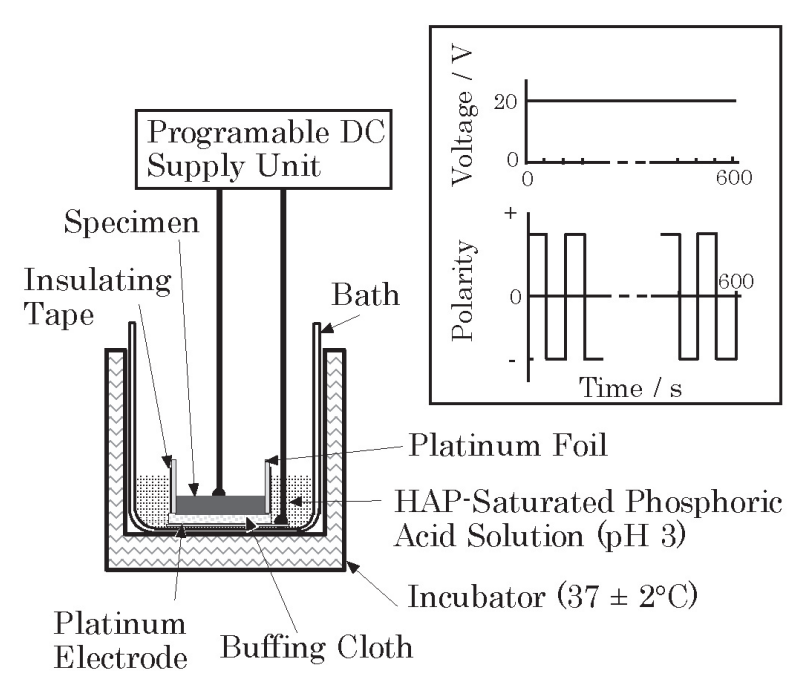

Fig. 1 Experimental setup

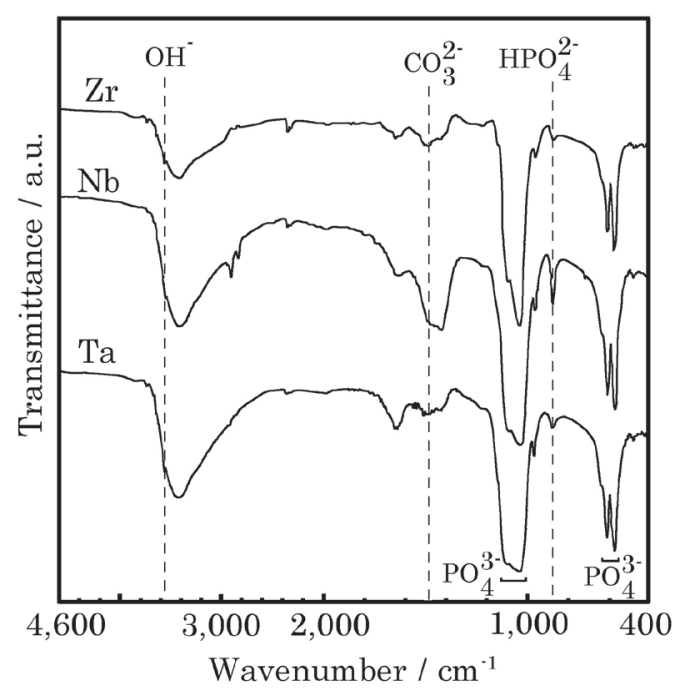

Fig. 2 FTIR spectra of the deposits
(TegraPol-11, Struers, Ballerup, Denmark). The specimens were ultrasonically cleaned in distilled water for $300 \mathrm{~s}$.

The experimental setup is illustrated in Fig. 1. HAP (HAP-200, Taihei Chemical Industrial Co., Ltd., Osaka, Japan) and phosphoric acid, $\mathrm{H}_{3} \mathrm{PO}_{4}$, (Wako Pure Chemical Industries, Osaka, Japan) were used for electrolyte preparation. Since HAP dissolves readily in acid solution, the $\mathrm{pH}$ of the saturated $\mathrm{HAP}-\mathrm{H}_{3} \mathrm{PO}_{4}$ solution for electrolyte preparation was adjusted to 3 with $\mathrm{H}_{3} \mathrm{PO}_{4}$. Platinum foil with an insulating tape was wrapped around the circumference of the specimen. A buffing cloth (Selvyt \#40-7008, Buehler) with a $0.3 \mathrm{~mm}$ thickness was inserted between the specimen and the platinum disk as an electrode. This sandwich assembly was immersed in $10 \mathrm{~mL}$ of the electrolyte solution maintained at $37 \pm 2{ }^{\circ} \mathrm{C}$ in an incubator (NDC-100, Nissin, Tokyo, Japan). A constant VDC of 20 was applied between the electrodes for $600 \mathrm{~s}$ via a programmable DC power unit (6633B, Agilent, CA, USA). The polarity of each electrode was automatically alternated between positive (or negative) and negative (or positive) every $60 \mathrm{~s}$. Finally, the treated specimens were allowed to dry in air at room temperature. A symbol of an element of the valve metal was used to identify the treated specimen.

\section{Surface characterizations}

The deposit and underlying substrates were compositionally and morphologically analyzed by Fourier transform infrared spectroscopy (FTIR) (FT/IR7300, Jasco, Tokyo, Japan), X-ray diffraction (XRD) (Assembly, Rigaku, Tokyo, Japan), X-ray photoelectron spectroscopy (XPS) (Quantum2000, ULVAC-PHI, Chigasaki, Japan), and atomic force microscopy (AFM) (SPM-4210, JEOL, Tokyo, Japan).
Fourier transform infrared spectroscopy

The deposited powder was scraped off from the treated surface, mixed with high purity $\mathrm{KBr}$ powder (Jasco, Tokyo, Japan), and compacted into a pellet form. The sample was scanned from 4,600 to $400 \mathrm{~cm}^{-1}$ under a 4 $\mathrm{cm}^{-1}$ resolution and the average of 500 scans was computed. The absorbance at the maximum peak in the specimen was about $10 \%$.

\section{$X$-ray diffraction analysis}

The deposited powder was analyzed using XRD, $\mathrm{Cu}$ target, $40 \mathrm{kV}, 30 \mathrm{~mA}$, scanning speed $1 \%$ min. X-ray diffraction patterns were indexed according to the structural data of Powder Diffraction File (ICDD, PA, USA, 1998).

\section{$X$-ray photoelectron spectroscopy}

XPS spectra were obtained using a spectrometer with $\mathrm{Al} \mathrm{K} \alpha$ radiation generated under $25 \mathrm{~W}$ at $15 \mathrm{kV}$. The peak positions, shifted by the electric charge accumulated in the insulating sample, were corrected by referencing the position of the $\mathrm{C} 1 \mathrm{~s}$ peak. Argon ion flux was employed to sputter the surface for $60 \mathrm{~s}$. Binding energies were calculated by curve fitting treatment using MultiPak software installed in the XPS analysis system. The $\mathrm{Ca} / \mathrm{P}$ atomic ratio was also calculated using the same software.

\section{Atomic force microscopy}

For the observation of nanometer scale topography, the treated specimens were ultrasonically rinsed in distilled water for $300 \mathrm{~s}$ and then allowed to dry in air at room temperature. The interface between the bulk and deposited powders was observed using a scanning probe microscope in the $\mathrm{AC}$ mode, which operates in the 
intermittent contact regime or in the non-contact regime. The lateral scan frequency of $160 \mathrm{kHz}$ resulted in a total recording time of $20 \mathrm{~min}$ per image. All images shown represent raw data of height images with no filtering.

\section{RESULTS}

Fourier transform infrared spectroscopy

The FTIR spectra of the deposits on the valve metals are shown in Fig. 2. The bands at about 3,600 and 630 $\mathrm{cm}^{-1}$ in the spectra came from $\mathrm{OH}^{-}$ions ${ }^{13)}$, whereas those at around 1,100 and $600 \mathrm{~cm}^{-1}$ originated from $\mathrm{PO}_{4}^{3-13)}$. The bands with weak intensity at around 1,450 $\mathrm{cm}^{-1}$ in the spectra were attributed to the components of the trace amount of $\mathrm{CO}_{3}{ }^{2-13)}$, while that at about 900 $\mathrm{cm}^{-1}$ came from $\mathrm{HPO}_{4}{ }^{2-}$ ions ${ }^{13)}$. Accordingly, the deposits were identified to be similar to CAPs with additional bands ascribed to impurity ion $\left(\mathrm{CO}_{3}{ }^{2-}\right.$ ion $)$.

$X$-ray diffraction analysis

The representative XRD patterns of the precipitated powder are shown in Fig. 3. The observed positions of diffraction lines were in agreement with the corresponding values for HAP $\left(\mathrm{Ca}_{10}\left(\mathrm{PO}_{4}\right)_{6}(\mathrm{OH})_{2}\right.$, JCPDS 84-1998) in all specimens and additional peaks were detected in $\mathrm{Zr}$ and $\mathrm{Nb}$. These additional peaks corresponded to DCPA ( $\mathrm{CaHPO}_{4}$, JCPDS 71-1759). Moreover, DCPD $\left(\mathrm{CaHPO}_{4} \cdot 2 \mathrm{H}_{2} \mathrm{O}\right.$, JCPDS 11-0293) was detected in $\mathrm{Nb}$. The diffraction pattern showed broadened and convoluted peaks.

\section{$X$-ray photoelectron spectroscopy}

The spectra of the surface survey scans of the deposited powders were similar except for the difference in the relative intensities of the peaks for the different

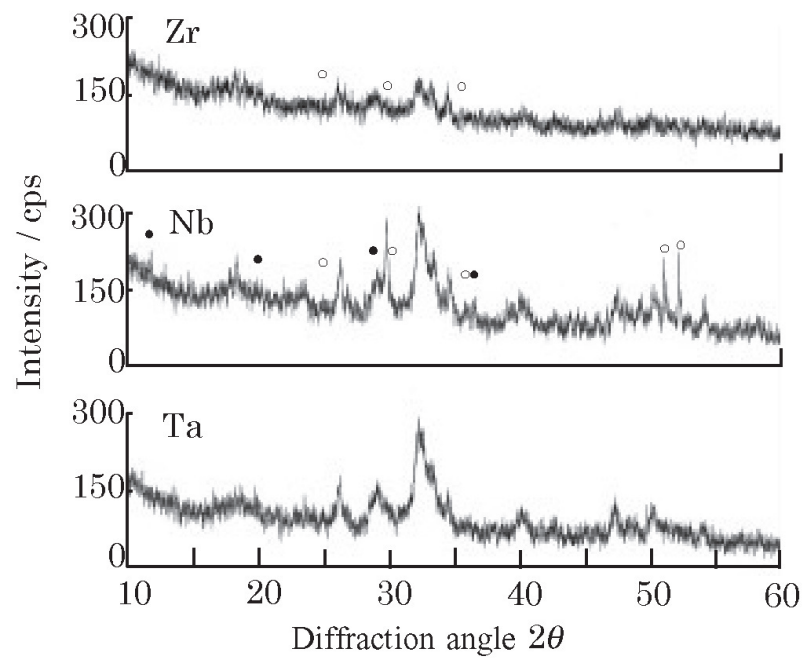

-: DCPA, $\mathrm{CaHPO}_{4} \cdots:$ DCPD, $\mathrm{CaHPO}_{4} 2 \mathrm{H}_{2} \mathrm{O}$ No symbol: HAP, Ca10 $\left(\mathrm{PO}_{4}\right)_{6}(\mathrm{OH})_{2}$

Fig. 3 XRD diffraction patterns of the deposits

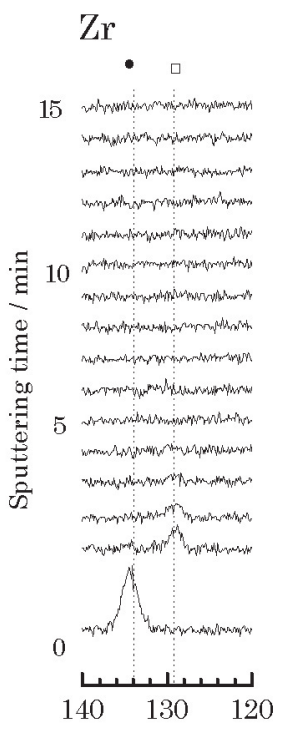

$\mathrm{Nb}$

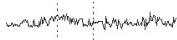

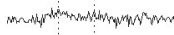

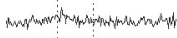

stem wam

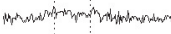

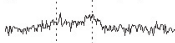

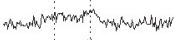

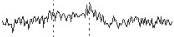

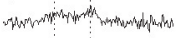

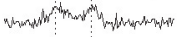

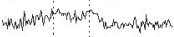

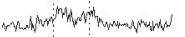

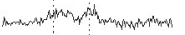

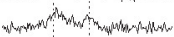

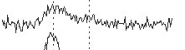

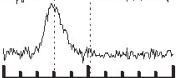

$\mathrm{Ta}$

- 口

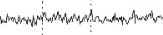

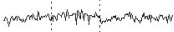

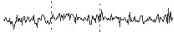

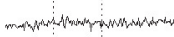

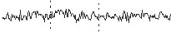

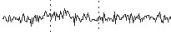

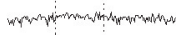

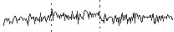

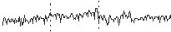

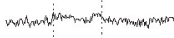

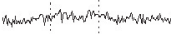

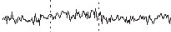

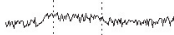

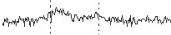

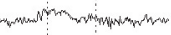

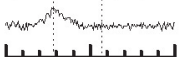

$\begin{array}{lll}140 & 130 & 120\end{array}$

Binding energy / eV

- $: \mathrm{P}^{5+}$ phosphate ion $\quad \square: \mathrm{P}^{3+}$ phosphate ion

Fig. 4 Depth profiles of the specimens by XPS

Table 1 Binding energy of the deposits and chemical reagent CAP

\begin{tabular}{|c|c|c|c|c|c|c|}
\hline & & \multicolumn{5}{|c|}{ Binding energy /eV } \\
\hline & & \multicolumn{5}{|c|}{ Specimen } \\
\hline & & $\mathrm{Zr}$ & $\mathrm{Nb}$ & $\mathrm{Ta}$ & HAP & DCPD \\
\hline Ca $2 p 3 / 2$ & $\mathrm{Ca}^{2+}$ & $346.74(2.42)$ & $346.79(1.92)$ & $346.67(1.99)$ & $346.3(1.72)$ & $346.7(1.81)$ \\
\hline Ca $2 p 1 / 3$ & & $350.31(1.68)$ & $350.34(1.68)$ & $350.20(1.68)$ & $349.9(1.53)$ & $350.3(1.53)$ \\
\hline P 2p3/2 & $\mathrm{PO}_{4}{ }^{2-}$ & $132.69(1.81)$ & $132.66(1.91)$ & $132.43(1.96)$ & $132.3(1.68)$ & $132.7(1.89)$ \\
\hline P $2 \mathrm{p} 1 / 3$ & & $133.72(1.48)$ & 133.92(1.63) & 133.67(1.75) & 133.1(1.81) & $133.7(1.81)$ \\
\hline \multirow[t]{3}{*}{$\mathrm{O} 1 \mathrm{~s}$} & $\mathrm{O}^{2-}$ & $530.27(1.97)$ & $530.43(1.81)$ & $530.32(1.87)$ & $530.2(1.67)$ & $530.4(1.89)$ \\
\hline & $\mathrm{OH}^{-}$ & $531.27(1.80)$ & $531.62(1.80)$ & $531.27(1.80)$ & $531.4(1.00)$ & $531.5(2.29)$ \\
\hline & $\mathrm{H}_{2} \mathrm{O}$ & $532.04(1.43)$ & $532.91(1.31)$ & $532.66(1.42)$ & $532.4(1.29)$ & $533.0(1.34)$ \\
\hline
\end{tabular}



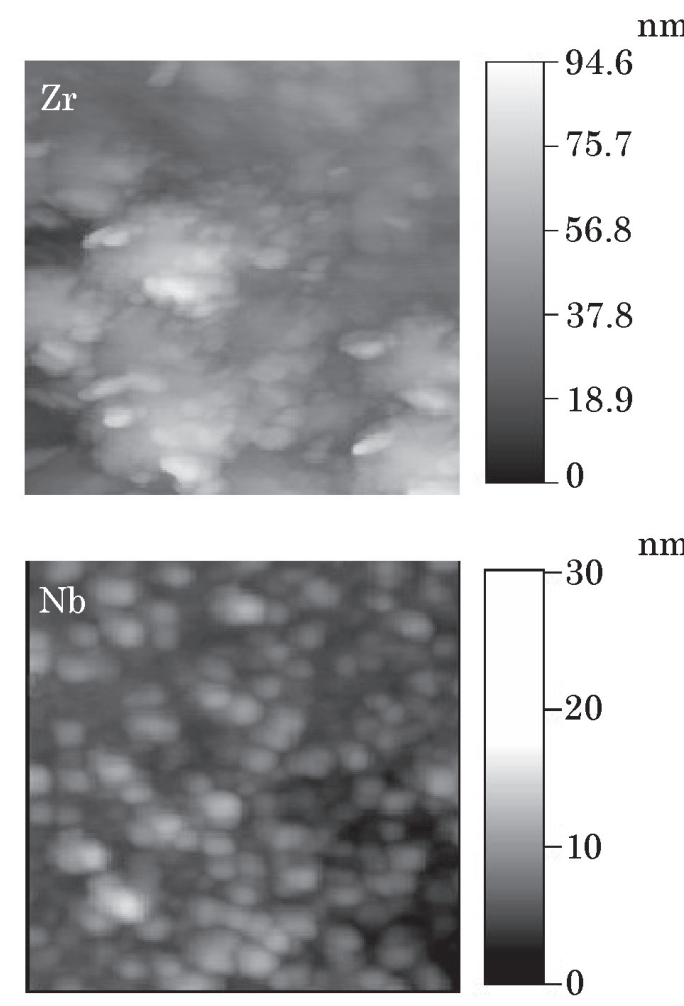

$\mathrm{nm}$

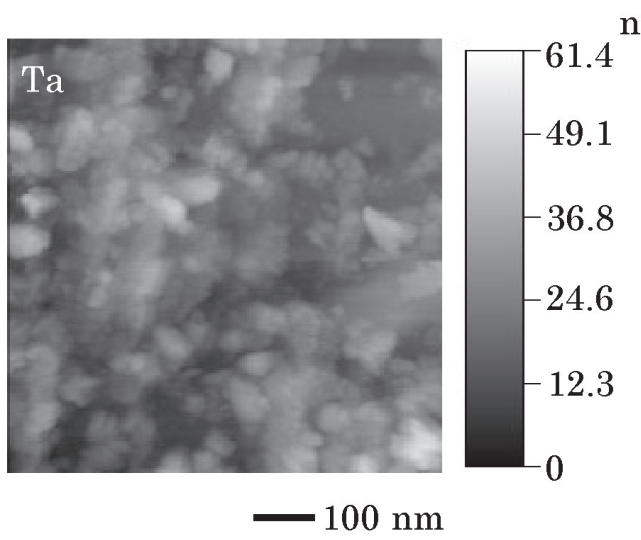

Fig. 5 AFM images of the deposits

elements. In addition to $\mathrm{Ca} 2 \mathrm{p}, \mathrm{P} 2 \mathrm{p}$, and $\mathrm{O} 1 \mathrm{~s}, \mathrm{C} 1 \mathrm{~s}$ was also found on the surface of all specimens in significant amounts. The binding energies calculated by the curve fitting treatment are shown in Table 1. The binding energies were mostly attributed to the chemical states of $\mathrm{Ca}, \mathrm{P}$, and $\mathrm{O}$ elements in the HAP and DCPD reagent matrixes. The $\mathrm{Ca} / \mathrm{P}$ atomic ratio of the deposits was in the range of $1.3-1.5$, which was significantly lower than that of a pure HAP.

The XPS depth profiles of the specimens are shown in Fig. 4. P $2 p$ XPS spectra before sputtering were observed at around $134 \mathrm{eV}$, and identified as $\mathrm{P}^{5+}$ due to $\mathrm{PO}_{4}^{3-14)}$. The peaks on $\mathrm{Zr}, \mathrm{Nb}$ and $\mathrm{Ta}$ were due to a decreased in $\mathrm{P}^{5+}$ with sputtering time, and were finally not observed on the specimens sputtered for 120, 480 and $160 \mathrm{~s}$, respectively. In contrast, a new peak at around $129.5 \mathrm{eV}$ appeared and its intensity increased with sputtering time, although this new peak was not observed on $\mathrm{Zr}, \mathrm{Nb}$ and Ta after sputtering for 300, 780 and $540 \mathrm{~s}$, respectively.

\section{Morphological analysis}

Representative AFM images showing the morphology of the deposited particles are shown in Fig. 5. Blocklike particles of about $50 \mathrm{~nm}$ length were observed on Ta. Granular composites with an average of $50 \mathrm{~nm}$ diameters were detected on $\mathrm{Nb}$. Ellipsoidal particles of about $200 \mathrm{~nm}$ length span and slender particles of 100 $\mathrm{nm}$ length span were observed on $\mathrm{Zr}$.

\section{DISCUSSION}

The deposit obtained using the new anodic oxidation method in the saturated $\mathrm{HAP}-\mathrm{H}_{3} \mathrm{PO}_{4}$ solution was found to be an aggregation of a few kinds of CAP. The crystallites were very small or poorly crystallized due to the diffraction pattern. XRD analysis data showed that the synthesized HAP on the valve metals was not strictly pure. In addition, DCPA and DCPD were detected in the deposits. These observations should explain the observed tendency of HAP to lower the $\mathrm{Ca} /$ $\mathrm{P}$ atomic ratio compared with the stoichiometric HAP. No peaks of valve metal oxide were found in XRD analysis data. Therefore, the valve metal oxide formed by this new anodic oxidation method would have amorphous nature. Here, the XRD analysis was important for phase investigation. On the other hand, the FTIR spectroscopic data compensated for some features that could not be observed by XRD analysis. A $\mathrm{HPO}_{4}{ }^{2-}$ band was found in the spectra of Ta. Therefore, it was assumed that $\mathrm{Ca}$ ions were lacking in the deposited HAP. Moreover, the $\mathrm{Ca} / \mathrm{P}$ ratio obtained on $\mathrm{Ta}$ by XPS $(\mathrm{Ca} / \mathrm{P}=1.3)$ supports this finding. Accordingly, the deposit on Ta was nonstoichiometric and was in agreement with a Ca-deficient HAP. On the other hand, the deposits on $\mathrm{Nb}$ and $\mathrm{Zr}$ included not only HAP but also DCPD and DCPA, respectively. However, we were unable to confirm whether their deposits were stoichiometric or nonstoichiometric. By the observations of AFM, the block-like, granular and ellipsoidal particles in the order of nanometer were observed on different metals. However, the particles were not morphologically distinguished in the crystal.

In general, when a valve metal is anodized, the electric current firstly runs through the experimental circuit, after which it decreases rapidly, and then finally hardly runs. This is because the oxide film formed on the valve metal has the properties of an ntype semiconductor ${ }^{15)}$. In this study, the electric current ran continuously because we employed the alternate polarity inversion method and a newly designed sandwich electrode. Tanaka et al. ${ }^{16)}$ reported that CAP was formed on Ti in Hanks' solution by low voltage (1-2 V) electrolytic treatments. Being different from our 
alternate polarity inversion method, they applied a cyclic potential with a trigonometric wave form at 1 or $10 \mathrm{~Hz}$ and inferred the following points. The cyclic exchange of anodic and cathodic currents might enhance the adsorption of $\mathrm{P}$ and $\mathrm{Ca}$ ions alternately. Also, the cathodic current might be a factor causing the high $\mathrm{Ca} / \mathrm{P}$ ratio of $\mathrm{CAP}$. The current was not examined and dissected in our study. It was assumed that the current affected the characterization of the deposited CAPs.

The oxide film was formed by anodization on the specimen when the specimen was a positive electrode (i.e., anode). Simultaneously, $\mathrm{O}_{2}$ was generated on the platinum foil wrapped around the specimen by water electrolysis. Moreover, $\mathrm{PO}_{4}{ }^{3-}$ ions moved near the electrode; thus, the concentration of the ions around the electrode is expected to be higher than that of the surroundings. On the other hand, $\mathrm{H}_{2}$ was generated on the opposite electrode (negative electrode; i.e., cathode). When polarity was inversed, the specimen became negative. $\mathrm{H}_{2}$ was generated on the platinum foil wrapped around the specimen, and $\mathrm{Ca}^{2+}$ ions may be predominant around the foil and specimen. These repeated process caused an increase in the concentration of $\mathrm{PO}_{4}{ }^{3-}$ and $\mathrm{Ca}^{2+}$ ions near the electrode. Therefore, the product of the concentrations of these ions, i.e. solubility product, was larger than that of CAPs, and then CAPs precipitation might occur. Moreover, $\mathrm{pH}$ would change near the specimen because of increasing $\mathrm{OH}^{-}$concentration due to water electrolysis. The factor of the increase in the concentration of $\mathrm{PO}_{4}{ }^{3-}$ and $\mathrm{Ca}^{2+}$ ions, and $\mathrm{pH}$ would affect the nuclear formation and morphology of CAPs. Consequently, the alternate polarity inversion method employing a sandwich electrode should easily promote CAP deposition under an applied low voltage.

Many studies ${ }^{17-18)}$ on the mechanism of CAP deposition on Ti from a solution containing $\mathrm{Ca}^{2+}$ and $\mathrm{PO}_{4}{ }^{3-}$ ions have been reported. Some of the typical CAP forms reported are $\mathrm{HAP}$, TCP $\left(\beta-\mathrm{Ca}_{3}\left(\mathrm{PO}_{4}\right)_{2}\right)$, OCP $\left(\mathrm{Ca}_{8} \mathrm{H}_{2}\left(\mathrm{PO}_{4}\right)_{6} 5 \mathrm{H}_{2} \mathrm{O}\right)$ and DCPD in order of increasing solubility ${ }^{19)}$. Different CAPs will form depending mainly on the $\mathrm{pH}$ of the solution during CAP precipitation ${ }^{19}$. Therefore, $\mathrm{pH}$ was crucial for determining the structure and composition of the electrodeposition products on the electrode. According to the ratio of the phosphate family (i.e., $\mathrm{H}_{3} \mathrm{PO}_{4}, \mathrm{H}_{2} \mathrm{PO}_{4}^{-}$, $\mathrm{HPO}_{4}{ }^{2-}$ and $\mathrm{PO}_{4}{ }^{3-}$ ) and as a function of $\mathrm{pH}$, the concentration of the phosphate family depends on the $\mathrm{pH}$ of the solution ${ }^{20)}$. Because the $\mathrm{pH}$ of the electrolyte used in this study was 3 , the ratio of $\mathrm{H}_{3} \mathrm{PO}_{4} / \mathrm{H}_{2} \mathrm{PO}_{4}^{-}$was about $0.1 / 0.9$. Also, the number of $\mathrm{H}_{3} \mathrm{PO}_{4}$ species decreased as $\mathrm{pH}$ increased. A large number of the chemical species of $\mathrm{H}_{2} \mathrm{PO}_{4}^{-}$were present at about $\mathrm{pH}$, and this number decreased are around $\mathrm{pH} 5$, whereas the number of chemical species of $\mathrm{HPO}_{4}{ }^{2-}$ increased at around $\mathrm{pH} 5$. At a neutral $\mathrm{pH}$, the ratio of $\mathrm{H}_{2} \mathrm{PO}_{4}{ }^{-} / \mathrm{HPO}_{4}{ }^{2-}$ was $0.5 / 0.5$, that is, the amount of $\mathrm{H}_{2} \mathrm{PO}_{4}^{-}$and $\mathrm{HPO}_{4}{ }^{2-}$ species was equal.

As mentioned before, the specimen surface serving as the cathode reduced water to $\mathrm{H}_{2}$, that is, $\mathrm{H}_{2} \mathrm{O}+\mathrm{e}^{-} \rightarrow$
$1 / 2 \mathrm{H}_{2}+\mathrm{OH}^{-}$, and the presence of $\mathrm{OH}^{-}$ions resulted in an increase in local $\mathrm{pH}$. Hence, the stability of $\mathrm{HPO}_{4}{ }^{2-}$ was greatly increased compared with that of $\mathrm{H}_{2} \mathrm{PO}_{4}^{-}$. It was speculated that the most probable reaction taking place near the cathode was the conversion of $\mathrm{H}_{2} \mathrm{PO}_{4}^{-}$to $\mathrm{HPO}_{4}{ }^{2-}$, that is, $\mathrm{H}_{2} \mathrm{PO}_{4}^{-}+\mathrm{OH}^{-} \rightarrow \mathrm{HPO}_{4}{ }^{2-}+\mathrm{H}_{2} \mathrm{O}$. Referring to Monma's suggestion regarding the chemical reactions involved in electrodeposition ${ }^{21,22)}$, the schematic of the precipitation reactions on the valve metal are summarized as follows.

$$
\begin{aligned}
& \mathrm{H}_{2} \mathrm{O}+\mathrm{e}^{-} \rightarrow 1 / 2 \mathrm{H}_{2}+\mathrm{OH}^{-} \\
& \mathrm{H}_{2} \mathrm{PO}_{4}^{-}+\mathrm{OH}^{-} \rightarrow \mathrm{HPO}_{4}^{2-}+\mathrm{H}_{2} \mathrm{O} \\
& \quad \mathrm{Ca}^{2+}+\mathrm{HPO}_{4}^{2-}+2 \mathrm{H}_{2} \mathrm{O} \rightarrow \mathrm{CaHPO}_{4} 2 \mathrm{H}_{2} \mathrm{O}(\mathrm{DCPD}) \\
& \quad \mathrm{Ca}^{2+}+\mathrm{HPO}_{4}{ }^{2-} \rightarrow \mathrm{CaHPO}_{4}\left(\mathrm{DCPA}^{2-}\right) \\
& \mathrm{HPO}_{4}{ }^{2-}+\mathrm{OH}^{-} \rightarrow \mathrm{PO}_{4}{ }^{3-}+\mathrm{H}_{2} \mathrm{O} \\
& 10 \mathrm{Ca}^{2+}+6 \mathrm{PO}_{4}{ }^{3-}+2 \mathrm{OH}^{-} \rightarrow \mathrm{Ca}_{10}\left(\mathrm{PO}_{4}\right)_{6}(\mathrm{OH})_{2}(\mathrm{HAP})
\end{aligned}
$$

Changes in these precipitation reactions depend on the concentrations of $\mathrm{OH}^{-}$and the phosphate family. Therefore, rapid precipitation of HAP would occur when a high $\mathrm{OH}^{-}$concentration on and near the valve metal would cause.

Based on the XPS depth profiles of the specimens (Fig. 4), $\mathrm{P}$ ions identified as $\mathrm{P}^{5+}$ due to $\mathrm{PO}_{4}{ }^{3-}$ were attributed to the outermost CAP. After sputtering, new peaks $(129.5 \mathrm{eV})$ attributed to $\mathrm{P}^{3+}$ appeared. The increase in the number of $\mathrm{P}^{3+}$ ions and that in the number of $\mathrm{P}^{5+}$ ions inside the film prepared using the new anodic oxidation methods were due to the anodic oxidation behaviors from the surface of the substrate in contact with the phosphoric acid solution. Nakahira et $a l{ }^{23)}$ reported that $\mathrm{P}^{5+}$ ions were mainly doped near the surface on a $\mathrm{TiO}_{2}$ film by anodic oxidation of $\mathrm{Ti}$ in phosphoric acid solution, and that $\mathrm{P}$ ions existed as $\mathrm{P}^{+3}$ ions inside the oxide films. Ferdjani et $a l^{24)}$ reported that a notable incorporation of phosphorus into the oxide layer occurred during its growth. Our results coincided with their results, though different metals were used in their experiment. The oxide film which incorporated phosphate ions was directly formed by anodic oxidation in the phosphoric acid solution. This characteristic was potentially important role for CAPs deposition.

In other words, a new functional film including phosphate ions would make a favorable environment condition for CAPs deposition. Furthermore, the $\mathrm{pH}$ of an electrolyte and the ratio of the phosphate family will affect the structure of the deposited crystal and its morphology. Nano-particles should be chemically active and must be the starting point in the formation of HAP in the body, a valve metal implant having the order of nano-deposit would be excellent for enhancing osteoconductivity.

\section{CONCLUSIONS}

A new method of anodization with alternate polarity inversion using a saturated $\mathrm{HAP}-\mathrm{H}_{3} \mathrm{PO}_{4}$ solution was applied to valve metals. The Ca-deficient HAP with a 
nano-grain size precipitated on Ta. The deposits on $\mathrm{Nb}$ and $\mathrm{Zr}$ were HAP, DCPD and DCPA, and HAP and DCPA, respectively. The XPS spectra of the specimens showed that phosphate ions were incorporated in the valve metal anodic oxide film. Thus, this surface characteristic had effects on the precipitation behavior of HAP, DCPD and DCPA. Moreover, the deposits on the valve metals had fine particles on the order of nanometers.

\section{ACKNOWLEDGMENT}

This work was supported by a Grant-in-Aid for Scientific Research by the Japan Society for the Promotion of Science, Scientific Research, (C) 18592116. The authors are grateful to Mrs. H. Honma, technician in the Biomaterial Science division, for her assistances in collecting references.

\section{REFERENCES}

1) Christoph L, Manfred P. Titanium ant titanium alloys: Fundamentals and applications, Wiley-VCH, Weinheim, 2003, pp. 425-427.

2) Ferracane J. Materials in dentistry, Lippincott Williams \& Wilkins, Baltimore Maryland, 2001, pp. 285.

3) Gladwin M, Bagby M. Clinical aspect of dental materials: Theory, practice, and cases. Lippincott Williams \& Wilkins, Baltimore Maryland, 2004, pp. 167-168.

4) Shibata K, Kamegai A. Titanium in dentistry: Biocompatibility of titanium. Quintessence, Tokyo, 1988. pp. 35-41.

5) Lacefield WR. Hydroxylapatite coatings. In: Hench LL, Wilson J, Editors, An introduction to bioceramics, World Scientific, Singapore, 1993, pp. 223-238.

6) Hanawa T, Kamiura Y, Yamamoto S, Kohgo T, Amemiya A, Ukai H, Murakami K, Asaoka K. Early bone formation around calcium-ion-implanted titanium inserted into rat tibia. J Biomed Mater Res 1997; 36: 131-136.

7) Kim HM, Miyaji F, Kokubo T, Nishiguchi S, Nakamura T. Granded surface structure of bioactive titanium metal prepared by chemical treatment. J Biomed Mater Res 1999; 45: 100-107.

8) Klein CPAT, Wolke JGC, Groot K. Stability of calcium phosphate ceramics and plasma sprayed coating. In: Hench LL, Wilson J, Editors, An introduction to bioceramics, World Scientific, Singapore, 1993, pp. 199-221.

9) Ishizawa $\mathrm{H}$, Ogino $\mathrm{M}$. Hydrothermal precipitation of hydroapatite on anodic titanium oxide films containing $\mathrm{Ca}$ and P. J Mater Sci 1999; 34: 5893-5898.

10) Ban S, Maruno S, Harada A, Hattori M, Narita K, Hasegawa J. Effect of temperature on morphology of electrochemicallydeposited calcium phosphates. Dent Mater J 1996; 15: 3138.

11) Monma H. Morphology and calcium-deficiency of electrochemically deposited apatite. Gypsum \& Lime 1993; 247: 427-433.

12) Hanna F, Hamid ZA. Electrodeposition of biomedical hydroxyapatite coating on titanium alloy substrate. Pigment \& Resin Tech 2003; 32: 319-325.

13) Markovic M, Fowler BO, Tung MS. Preparation and comprehensive characterization of a calcium hydroxyapatite reference material. J Res Natl Inst Stand Technol 2004; 109: 553-568.

14) Moulder JF, Stickle WF, Sobol PE, Bomben KD. Handbook of X-ray photoelectron spectroscopy. Phsical Electronics, Inc., Minnesota, 1995, pp. 233, 59.

15) Kubaschewski O, Hopkins BE. Oxidation of metals and alloy. Butterworths London Second Edition,1962, pp. 24.

16) Tanaka $Y$, Kobayashi E, Hiromoto S, Asami K, Imai H, Hanawa T. Calcium phosphate formation on titanium by low-voltage electrolytic treatments. J Mater Sci Mater Med 2007; 18: 797-806.

17) Lin S, LeGeros RZ, LeGeros JP. Adherent octacalciumphosphate coating on titanium alloy using modulated electrochemical deposition method. J Biomed Mater Res A 2003; 66: 819-828.

18) Kishida T. Thin hydroxyapatite coating prepared on a titanium plate by a two-stage discharging method in an electrolyte. J J Dent Mater 2001; 20: 77-86.

19) Chow LC. Solubility of calcium phosphates. In:Chow LC, Eanes ED, Editors. Octacalcium phosphate. Karger, Switzerland, pp. 94-111.

20) Day RA, Underwood AL: Quantitative Analysis, 1 ed, Baifukan, Tokyo, 1971, pp. 97-99.

21) Monma H. Electrochemical Deposition of Calcium-Deficient Apatite on Stainless Steel Substrate. J Ceram Soc Jpn 1993; 101: 737-739.

22) Monma H, Nemoto O, Takahashi S, Kobayashi H. Electrolytic Formation and Morphology of Biomimetic Apatite Coatings. J Electroceramics 1999; 4S1: 135-140.

23) Nakahira A, Konishi K, Yokota K, Honma T, Aritani H, Tanaka K. Synthesis and characterization of $\mathrm{TiO}_{2}$ doped with $\mathrm{P}$ ions by anodic oxidation of titanium in acid solution. J Ceram Soc Jpn 2006; 114: 46-50.

24) Ferdjani S, David D, Beranger G. Anodic oxidation of titanium in phosphoric acid baths: phosphorus incorporation into the oxide. J Alloys Compounds 1993; 200: 191-194. 